\title{
LA DETERMINACIÓN DE LA MERX PECULIARIS COMO ETAPA PREVIA A SU REPARTO*
}

\author{
Patricio Lazo**
}

RESUMEN: El presente trabajo centra su mirada en lo que podría considerarse la etapa previa del reparto de la merx peculiaris, es decir, de la tributio. Puesto que el objetivo de dicha etapa es la determinación de los bienes que entrarán en el reparto, el desafío de la jurisprudencia consistió en establecer criterios para ello. Esta labor se lleva a cabo a partir de la interpretación, en primer lugar, de unas pocas cláusulas edictales y, principalmente, merced a la constante revisión del aparato conceptual. El trabajo pone de relieve cómo dos herramientas conceptuales, a saber, la distinción de negocios relacionados con la merx peculiaris y la operacionalización de la subrogación real, permitieron a la jurisprudencia romana ofrecer un esquema útil para la configuración de la etapa que debía preceder al reparto.

PALABRAS CLAVE: Merx peculiaris - tributio - subrogación real - actio tributoria - acciones adiecticiae qualitatis

\section{THE DETERMINATION OF THE MERX PECULIARIS AS A PRELIMINARY PHASE TO ITS DISTRIBUTION}

ABSTRACT: This essay examines what might be called the previous phase of the distribution of the merx peculiaris, that is to say, the tributio. The challenge for the roman jurisprudence was to set up the criteria to identify the goods that will be distributed, considering that this is precisely the aim of this stage. First, this goal was reached by interpreting a few edictal clauses and mainly through a constant examination of concepts. The author stresses the way in which the concepts of differentiation of business related to the merx peculiaris and real subrogation, allowed roman jurisprudence to provide a useful scheme for configuring the phase that should precede distribution.

Este trabajo forma parte del proyecto de investigación Puente, código 162.705/2015, de la Pontificia Universidad Católica de Valparaíso, como asimismo del Proyecto Anillo-Conicyt, soc- 1111 .

Fecha de recepción: 4 de junio de 2015.

Fecha de aceptación: 26 de agosto de 2015.

** Doctor en Derecho y profesor de Derecho Romano de la Pontificia Universidad Católica de Valparaíso (CHILE). Correo: patricio.lazo@ucv.cl 
KEYWORDS: Merx peculiaris - tributio - real surrogation - actio tributoria-actiones.

Sumario: 1). Introducción. 2). La mercancia como objeto del reparto. 3). Ex ea merce. 4). La subrogación real. 5). Conclusiones.

\section{1) INTRODUCCIÓN}

La tributio es un mecanismo de reparto de los bienes gestionados por un sujeto a potestad; específicamente, ella concierne al esclavo que ha administrado un peculio ${ }^{1}$, masa de bienes dentro de la cual se diferencia otra $-\mathrm{u}$ otras, según el caso- denominada merx peculiaris (mercancía del peculio $)^{2}$. Esta última masa de bienes es aquella que queda afecta a la gestión de determinados negocios realizados por el esclavo, con el conocimiento -scientia- de su dueño ${ }^{3}$. Aunque no contamos referencias textuales que remitan inequívocamente a la idea de insolvencia, suele admitirse una relación de causa-efecto entre la que afectaría a la merx peculiaris y el reparto o tributio que se lleva adelante con los bienes que en ella subsisten ${ }^{4}$. Por lo demás, no existe duda de que un patrimonio especial como el peculio puede estar afecto a insolvencia 5 , de modo que, dentro de esa misma lógica, nada impediría que una masa de bienes como la merx pe-

1 Para un estudio sobre el uso de los esclavos en las actividades productivas, Buti, Ignazio (1976) Studi sulla capacità patrimoniale dei servi. Napoli: Jovene, 240 pp., p. 13 ss.; DI PorTo, Andrea (1984) Impresa collettiva e schivavo 'manager' in Roma antica. Milano: Giuffrè, 407 pp. Sobre la importancia del peculio en la vida económica romana, Kirschenbaum, Aaron (1987) Sons, slaves and freedmen in Roman commerce. Jerusalem - Washington D.C.: The Magna Press, The Hebrew University, The Catholic University of America Press, 244 pp.; KNOCH, Stefan (2005) Sklavenfürsorge im römischen Reich. Formen und Motive. Hildesheim - Zürich - New York: Georg Olms, 338 pp., p. 178. ss.

2 Di Porto (1984) 54, atribuye a la merx peculiaris un carácter autónomo, en cuanto patrimonio separado del peculio; por el contrario, Chiusi (1993) "Contributo allo studio dell'editto 'de tributoria actione"”. Atti della Accademia Nazionale dei Lincei, serie IX, vol. III, fasc. 4, pp. 283 - 397, p. 333 ss., después de un extenso recorrido por las concepciones que la doctrina romanística ha sostenido acerca de la naturaleza de la merx peculiaris, expresa su distancia respecto de tal predicamento.

3 SuÁrez Blásquez, Guillermo (2010) "El peculio como ente autónomo y matriz de la 'merx peculiaris”. Revista de Estudios Histórico-Jurídicos, vol. 32, pp. 119-125; Lazo, Patricio (2013) “La 'merx peculiaris' como patrimonio especial”. Revista de Estudios Histórico-Juridicos, vol. 35, pp. 179-191.

4 Aubert, Jean Jacques (2013) “Dumtaxat de peculio': What's in a Peculium or Establishing the Extent of the Principal's Liability". En Du Plessis, Paul J. (editor). New Frontiers. Law and Society in the Roman World. Edinburgh: Edinburgh University Press, 246 pp., p. 202, pone en relación la insolvencia con la actio tributoria, aunque sin profundizar.

5 Un estudio sobre la insolvencia del peculio puede verse en Kroppenberg, Inge (2001) Die Insolvenz im klassichen römischen Recht. Wien-Köln: Böhlau, 526 pp., p. 130 ss. 
culiaris pudiera exponerse a similar contingencia, de donde se sigue que el reparto vendría a constituir un procedimiento por medio del cual el acreedor consigue un pago proporcional a su acreencia, en el contexto de la insolvencia de su deudor ${ }^{6}$.

Luego, bajo la premisa de tratarse la tributio de un procedimiento de insolvencia, interesa en este trabajo centrar la mirada, en el contexto de la tematización que de este concepto hizo la jurisprudencia romana, sobre el problema de la determinación del objeto del reparto, es decir, de la suma de criterios conforme a los cuales se diferencian los bienes existentes en el peculio que podrán considerarse incluidos en dicho reparto, de aquellos que permanecerán ajenos. Como se podrá barruntar, una adecuada solución a este problema resultará no solo útil para la realización del reparto mismo, sino también para el análisis de los problemas derivados de la distribución dolosa, hipótesis importante -como se sabe- del ejercicio de la actio tributoria. Con todo, los problemas referidos a la ejecución del reparto y a las vicisitudes que podría experimentar, no son materia de este estudio.

\section{2) LA MERCANCÍA COMO OBJETO DEL REPARTO}

En cuanto procedimiento de insolvencia, la tributio consiste en un reparto de los bienes constitutivos de la merx peculiaris subsistente, después de la gestión que en su momento haya hecho el esclavo a cargo de ella. Por consiguiente, los interesados en concurrir al mismo son los acreedores que han gestionado actos o contratos con el esclavo a cargo de la merx peculiaris.

6 VAliño, Emilio (1967) “La 'actio tributoria”. Studia et Documenta Historiae et Iuris, vol. 33, pp. 103-128, p. 113, sostiene que la vocatio in tributum "supone, por lo tanto, un expediente voluntario de reparto, una especie de concurso de acreedores familiar” y, a continuación señala que ella "naturalmente, no sigue las normas del procedimiento ejecutivo, en cuanto que la vocatio no es la consecuencia de una acción, ni aparece en ella, ni en sus actos subsiguientes". Esta última afirmación de VAliño resume una de las tesis de su trabajo, en el sentido que la tributio precedería al ejercicio de la actio tributoria y no sería, por consiguiente, un efecto de esta. Esta tesis es contradicha por CHIUsi (1993) 350 ss., esp. 353, para quien es la actio tributoria la que provoca la tributio. En esta espinosa cuestión, en la que terció Domingo, Rafael (1994-1995) "Recensión a Tiziana J. Chiusi, Contributo allo studio dell'editto de tributoria actione". Seminarios Complutenses de Derecho Romano vol. 5 (Suplemento), pp. 56-64, p. 62 s., quien, armonizando ambas posturas, propone la existencia de dos acciones: una contractual y adyecticia, que sería aquella capaz de provocar la tributio, al tiempo que una segunda acción, penal e in factum, que sería aquella provocada por una dolosa distribución de la merx peculiaris; los juristas habrían hablado indistintamente de actio tributoria en uno y otro caso, sin reparar en la probable confusión. Se trata, en mi opinión, de una hipótesis posible y probable, cuya principal virtud es la de explicar de mejor forma las contradicciones -en definitiva, aparentes- en las fuentes, siempre aquejadas de fragmentarización. 
Un aspecto singular de esta concurrencia es que el dueño del esclavo puede ser uno de los concurrentes al reparto, en calidad de acreedor de aquel. Que el dueño fuese acreedor de su esclavo no tenía nada de extraño, dado el alto nivel de despersonalización que alcanzó la gestión del peculio en manos de los dependientes -hijo o esclavo- de un pater familias ${ }^{7}$. En sede de la actio de peculio, esta situación tenía su refrendo en el privilegio del pater o dominus de detraer del peculio, antes que los acreedores, aquello que le fuese adeudado; de hecho, la definición del peculio que habría ofrecido Tuberón se hace cargo de esta realidad, en cuanto restringe su composición a aquello que el esclavo tiene con permiso del dueño, una vez que se ha deducido lo que se le debe ${ }^{8}$. La tributio, en cambio, supone el fin de este privilegio, pero no del derecho del dominus de concurrir al reparto -que, por lo demás, lo llevará adelante él mismo, salvo que renuncie a hacerlo y el pretor deba nombrar a otro9 - eso sí, en igualdad de condiciones que el resto de los acreedores y excluida, por consiguiente, la posibilidad de detraer anticipadamente su parte ${ }^{10}$.

Los planteamientos en torno al objeto del reparto tienen una premisa obvia: lo que se ha de repartir son bienes que, formando parte del peculio gestionado por el esclavo, son diferenciables del resto, en tanto en cuanto forman parte de la masa denominada merx peculiaris. Sin embargo, parece evidente la insuficiencia de un planteamiento en los términos que se acaba de hacer, ya que la diferenciación entre aquellos bienes que componen el peculio y que se encuentran incluidos en la merx peculiaris y aquellos que no lo están, tiene una relevancia principalmente contable, puesto que no necesariamente los bienes constitutivos de la merx han sido separados de los demás del peculio. Además, que resulta obvio que desde la constitución del peculio hasta el momento del reparto ha transcurrido un espacio de tiempo. A ello se suma el hecho de que el esclavo habrá gestionado durante dicho período - todo indica que con poca fortuna- la merx peculiaris, de modo que la masa de bienes de la que hablamos habrá venido alterando su composición a lo largo del tiempo. Estos hechos darán pie para que los juristas romanos realicen distinciones y profundicen

7 Abatino, Barbara - Dari-Mattiacci, Giuseppe - Perotti, Enrico C. (2011) "Depersonalization of Business in Ancient Rome". Oxford Journal of Legal Studies, vol. 31, No 2, pp., 365-389.

8 D. 15,1,5,4 (Ulp. 29 ad ed.): Peculium autem Tubero quidem sic definit, ut Celsus libro sexto digestorum refert, quod servus domini permissu separatum a rationibus dominicis habet, deducto inde si quid domino debetur. En D. 15,1,4,5 se contempla, incluso, la posibilidad de que las deudas contraídas con el dominus agoten -siempre desde un punto de vista contable- el peculio, lo que no obsta que los bienes comprendidos en este continúen siendo considerados peculiares.

9 D. $14,4,7,1$.

10 Lazo, Patricio (2010) “El contexto dogmático de la 'par condicio creditorum' en el derecho romano”. Revista de Derecho, Universidad Católica del Norte, vol. 17, No 2), pp. 79-97. 
en el problema de la determinación exacta de los bienes que, estando en poder del esclavo y formando parte del peculio, deberán ser objeto del reparto. A este respecto, hay al menos dos herramientas conceptuales de las que se servirá la jurisprudencia para la determinación de los bienes que entrarán en el reparto o tributio y en los cuales quisiera centrar mi atención: de una parte, la distinción entre aquellos negocios que se refieren a la merx peculiaris y, en segundo término, la operacionalización de la subrogación real ${ }^{11}$.

\section{3) EX EA MERCE}

El edicto exige que el reparto recaiga sobre los bienes relacionados con las negociaciones para los cuales había sido entregada la mercancía. Si nos atenemos a la reconstrucción leneliana, una cláusula del edicto pretorio sería clave en este sentido: "si quid cum eo eius mercis nomine contractum erit, eius, quod ex ea merce erit eove nomine receptum erit"12. Conforme a esta reconstrucción, existiría una referencia a aquellos que podrán concurrir al reparto o distribución de la merx y, junto con ello, una indicación de las causas por las cuales estos podrían concurrir. Las cláusulas transcritas (separadas por una coma) establecerían, como primera causa, "aquellas obligaciones que se hubiesen contraído con el esclavo (quid cum eo... contractum erit) en nombre de su mercancía (eius mercis nomine)", en tanto que la segunda haría referencia a los bienes adquiridos en virtud de la gestión de la mercancía, así como a la mercancía como tal (ex ea merce). Por consiguiente, de acuerdo con la primera de las cláusulas, quedarían excluidos de concurrir al reparto los acreedores del esclavo que no hubiesen generado con este una relación obligatoria cuyo objeto fuese dicha mercadería.

La necesidad de que las negociaciones que realizaría el esclavo debiesen estar referidas a la merx peculiaris se puede advertir en varios pasajes. Así, por ejemplo, Ulpiano expresa en D. 14,4,5, que al reparto debe entrar "todo aquello que a causa de la mercancía y aquello que a causa de lo debido a ella se ha recibido"13. Asimismo, el mismo jurista, sostiene en D. 14,4,5,12: Sed et si adhuc debeatur mercis nomine a quibusdam, quibus

11 Un estudio completo sobre la subrogación real en los patrimonios especiales es el de ANDRÉs Santos, Francisco (1997) La subrogación real en los patrimonios especiales. Valladolid: Secretariado de Publicaciones e Intercambio Científico de la Universidad de Valladolid, 317 pp.

12 Lenel, Otto (1927) Das Edictum Perpetuum. 3a ed. Leipzig: Tauchnitz, 579 pp., p. 271.

13 D. 14,4,5,5 (Ulp. 29 ad ed.): Per hanc actionem tribui iubetur, quod ex ea merce et quod eo nomine receptum est. Según ANDrÉs SANTOs (1997) 202 s., la expresión mercis nomine "podría referirse a lo que se debe al sometido a raíz de los negocios relacionados con la merx, pero que aún no se le ha satisfecho, es decir, los créditos de que dispone el subiectus contra el deudor con quien usualmente realiza negocio con medios de la merx"; SuÁrez BLÁzquez, Guillermo (2013) "Concurso mercantil de acreedores: 'vocatio in tributum'. La acción tribu- 
solebat servus distrahere, hoc quoque tribuetur, prout fuerit receptum [Si por causa de la negociación de la mercancía se debiera todavía alguna cosa por aquellos a los que el esclavo acostumbraba a vender, igualmente se entregará esto si se hubiese cobrado]. De modo similar, el mismo Aunque, en primer término este último responsum tiene más bien por finalidad decidir si pueden repartirse o no aquellos incrementos que, aunque devengados, no hubiesen sido aún enterados al peculio, por el hecho de ser debidos por los deudores, se hace ahí una referencia al negocio que se ha hecho respecto de la mercancía y se refiere, precisamente, a esos bienes. Sin perjuicio de lo que se dirá más adelante sobre la subrogación real, interesa aquí la respuesta de Ulpiano desde la perspectiva de la delimitación que impone el que los bienes a repartir se procedan de aquellos negocios realizados con la mercancía o en nombre de ella.

La cláusula edictal antes citada aparece también en otros edictos, como los de institoria y exercitoria actione, aunque con una redacción ligeramente diferente: no se hace en ellas referencia a la merx, sino a la res, es decir, a los negocios que se habría encomendado a los dependientes respecto de los cuales existiría una praepositio. Por ahora no interesa conocer en profundidad el régimen que atañe a las acciones institoria y exercitoria, sino explicitar que la cláusula en análisis forma parte de una panoplia de nociones que constituyen parte significativa del régimen de ciertas acciones adyecticias y que, además, serán desarrollados por la interpretación de los prudentes. En este orden de cosas, la delimitación de la concurrencia al reparto solo en virtud de negocios cuyo objeto fuese la mercancía tiene, desde el punto de vista dogmático, dos aspectos cuya respuesta no es en absoluto obvia. La primera de estas cuestiones es el significado que ha de atribuirse en este edicto a la voz merx. La segunda es acerca de qué ocurre cuando un esclavo se dedica a diversos negocios y tiene, por consiguiente, diferentes acreedores. Ambos problemas, como se verá a continuación, no vienen resueltos por la cláusula edictal, sino que su solución debe ser afrontada por la jurisprudencia romana. Comparadas con las soluciones que acaban de examinarse, las que tienen relación con estos otros problemas ponen en juego la integración de elementos diferentes a aquellos que se presentan a propósito de la interpretación antes vista.

La voz merx tendría, en principio, el significado de aquellas cosas que se compran o venden. Así lo pone de manifiesto Ulpiano, cuando opone la acepción amplia que daría Pedio a este concepto, como se lee en D.14,4,1,1: Licet mercis appellatio angustior sit, ut neque ad servos fullones vel sarcinatores vel textores vel venaliciarios pertineat, tamen Pedius libro quinto decimo scribit ad omnes negotiationes porrigendum edictum [Aunque

toria frente al dueño (empresario corrupto)". Revista de Derecho, Universidad del Norte vol. 39, pp. 173-197, p. 189. 
la denominación de mercancía sea tan estricta que no comprenda a los esclavos bataneros, sastres, tejedores y revendedores de esclavos, sin embargo, escribe Pedio en su libro 15 <digestos> que se ha de extender por este edicto a toda clase de negociación]. La cuestión acerca del sentido que debemos dar al fragmento de Ulpiano no es del todo pacífica. De una parte, podría argumentarse que en él Ulpiano deja entrever un cierto agotamiento del concepto de merx peculiaris, desde una acepción restringida, que da paso a otra de mayor amplitud, que sería la postulada por Pedio. De este modo, mientras en la acepción restringida la noción de merx solo habría designado las cosas venales, a partir de Pedio ella habría, además, servido para designar los servicios que prestaría un batanero o un sastre, $y$, en definitiva, para designar a toda clase de negociación. Una tesis en sentido opuesto ha sostenido Chiusi, para quien la noción defendida por Pedio no habría modificado el concepto de merx, sino que, más bien, habría servido para fundar la concesión de las acciones exemplo tributoriae y quasi tributoria, que habrían ido concediéndose caso a caso ${ }^{14}$. En nuestra opinión, esa como ejemplificación de servicios a la que hace referencia Ulpiano cuando habla de la existencia de más de una merx gestionada por el esclavo ${ }^{15}$, hablaría a favor de la hipótesis del triunfo de Pedio, en el sentido de una genuina extensión del concepto de merx ${ }^{16}$. Las razones para que Pedio haya impulsado una tan significativa transformación del significado, hablaría, en primer término, de la conveniencia para su época de la elaboración de una acepción dogmática del término, dotada de un mayor nivel de abstracción y, en segundo lugar, de un muy probable éxito del procedimiento de reparto, en el sentido de validarse como un expediente efectivo. Por lo anterior, creo más que en este punto podemos dar por establecido que, aunque la original significación de merx tuvo que ver con la compra y venta de distintas clases de bienes, la jurisprudencia clásica habría operado una nueva comprensión del mismo sustantivo, que habría servido, ya no solo para designar aquellos bienes destinados a comprar o vender, sino también para designar los bienes que sirven de base a la prestación de servicios ${ }^{17}$.

14 Chiusi (1993) 314 ss., basa su tesis en las exégesis que dedica a D. 14,4,1,1 (a contar de p. 314 ss.); 14,1,1,19-20 (p. 321 ss.) y D. 14,1,6 pr. (p. 328 ss.).

15 Los ejemplos se recogen en D.14,4,5,15, pasaje en el cual Ulpiano refiere que un esclavo se dedica al negocio de trajes y al de tejidos. Podría tratarse, ciertamente, de la confección de trajes y, en el segundo caso, de la venta de tejidos, ejemplos que recogerían las dos posibilidades de consideración de la merx, tanto como bienes (tejidos), como servicios (confección).

16 Por el contrario, Giachi, Cristina (2005) Studi su Sesto Pedio. Milano: Jovene, 601 pp., p. 388, piensa que es poco probable que la proposición de Pedio haya alcanzado éxito y conjetura, a partir de D. 14,4,5,4, que la jurisprudencia debió mantenerse en una posición restrictiva; VALIÑo (1967) 110.

17 Cabe argumentar que, al menos para el ejercicio de la banca, podría haber adoptado como estructura jurídica la merx peculiaris. Un indicio en este sentido lo ve De Churruca, Juan (1991) "La quiebra de la banca del cristiano Calisto". Seminarios Complutenses de Derecho 
El segundo problema, esto es, el relativo a la pluralidad de negocios o de acreedores, pone en juego otros, relacionados esta vez con la determinación del activo. Aquí se advierte la utilización, aunque de forma tenue, de criterios contables. Quizá la causa de que no aparezcan desarrollados con mayor laxitud dichos criterios se relacione con los problemas ante los cuales ellos son utilizados, los que se reducen, en el supuesto que se analizará, a impedir la confusión patrimonial. Como veremos a continuación, uno de los pasajes utiliza precisamente la figura del contador de uno de los negocios basados en una merx peculiaris para referirse al problema, lo que atestigua fehacientemente el uso de instrumentos de contabilidad ${ }^{18}$.

Como adelantábamos, el problema principal tendría lugar ante la pluralidad de acreedores y, al mismo tiempo, de mercaderías peculiares, ya que se plantearía el problema de si todos participarían de todas las mercaderías, o bien, se haría de forma distinta. Este supuesto es el que aborda Ulpiano, en dos fragmentos (D. 14,4,5,15-16) ${ }^{19}$, ofreciendo des-

Romano, vol. 3, pp. 61-86, que refiere la situación de Calisto, esclavo de Carpóforo, ambos cristianos -el primero llegaría a ser Papa y santo-, narrada en Hipólito de Roma, Refutación de todas las herejias, 9,12,1-13. Los hechos, ocurridos a fines del s. II, consisten en que Carpóforo confía a su esclavo Calisto, a instancias de este, una importante suma de dinero para que este lo dedique a la actividad bancaria. En el ejercicio de esta, Calisto recibe depósitos que luego malgasta, viéndose imposibilitado de devolverlos; según el texto -la traducción corresponde a De Churruca- Calisto "se encontraba en dificultades" (Refut. omn. haer. $9,12,1$ i.f.), lo que significaba su quiebra. Con ello, no solo perjudica a los depositantes, sino también el buen nombre de Carpóforo. De Churruca entiende que las posibilidades podrían ser que, o bien Carpóforo entregó un peculio a Calisto para que realizase un negocio, con conocimiento o sin aprobación del primero, o bien lo puso como institor al frente de un negocio de banca. En el primer caso, y a partir del supuesto de la configuración de la merx peculiaris, habría sido posible el reparto de los bienes que quedaban del peculio, situación que, finalmente, no ocurrió. Petrucci, Aldo (1991) Mensam exercere. Studi sull'impresa finanziaria romana, Napoli: Jovene, 418 pp., p. 367, a su vez, ante la alternativa de conjeturar un peculio o una praepositio institoria, cree más probable la gestión del primero por parte de Calisto. Además, aunque estima difícil de descifrar la posición subjetiva de Carpóforo, excluye la ignorancia y, aunque no ve claro si la concessio peculii refleja voluntas o mera scientia, cree que hay razones para postular la primera. Ello trae como consecuencia la eventual procedencia de la actio de peculio, contra De Churruca, que la descarta (p. 75 ss.). Al igual que este último, Petrucci (p. 391) ve un caso claro de insolvencia. Para un panorama de la actividad bancaria, además de la obra de Petrucci ya indicada, Cerami, Pietro - Petrucci, Aldo (2010) Diritto commerciale romano. Profilo storico. 3a ed. Torino: Giappichelli, 337 págs., p. 109 ss.

18 Sobre la actividad contable en los negocios romanos, Giménez Barriocanal, Fernando (2003) La actividad económica en el Derecho romano. Análisis contable. Madrid: Dykinson, 454 pp., p. 45 ss.

19 D. 14,4,5,15: Si plures habuit servus creditores, sed quosdam in mercibus certis, an omnes in isdem confundendi erunt et omnes in tributum vocandi rell? [Si el esclavo tenía varios acreedores, pero algunos relativos a determinadas mercancías, ¿se habrán de confundir todos sin distinción de mercancías y habrán de ser llamados todos al mismo reparto ut puta duas negotiationes exercebat, puta sagariam et linteariam, et separatos habuit creditores. puto separatim eos in tributum vocari: unusquisque enim eorum merci magis quam ipsi credidit] D. 14,4,5,16: Sed si duas tabernas eiusdem negotiationis exercuit et ego fui tabernae verbi gratia quam ad Bucinum habuit ratiocinator, alius eius quam trans Tiberim, aequissimum puto separatim tri- 
de luego una sola respuesta. Se sigue de ambos que no puede dudarse de la imposibilidad de llamar a todos los acreedores al reparto de una misma mercadería ${ }^{20}$. Con todo, el segundo ejemplo tiene la particularidad de que el acreedor ha sido el contador (ratiotinator) de uno que tenía dos tiendas en lugares diferentes. Entre ambos fragmentos aparece con claridad el fundamento de Ulpiano para su decisión: el primero y más importante de ellos es que es más justo diferenciar ambas masas, porque con ello se evita el perjuicio de los demás acreedores, ya que impide que unos hagan efectivos sus créditos en la masa de bienes en las que los otros han contraído sus respectivas obligaciones con el esclavo (aequissimum puto separatim tributionem faciendam, ne ex alterius re merceve alii indemnes fiant, alii damnum sentiant). Esta razón ofrecida por Ulpiano presupone una contabilidad que permita hacer efectiva esta diferenciación patrimonial. Solo sobre esta base Ulpiano podrá afirmar que se es acreedor de la mercadería más que del esclavo (enim eorum merci magis quam ipsi credidit); la contabilidad por separado de cada mercadería peculiar se justifica, por consiguiente, en razón de aquello que se ha dado en llamar una "despersonalización" de los negocios romanos, al configurar la merx peculiaris como patrimonio separado dentro del peculio, patrimonio que concentra exclusivamente las consecuencias del incumplimiento de obligaciones contraídas en la gestión de ese peculio. La gestión contable de las mercaderías del peculio harán posible, además, que tanto el dueño como los demás acreedores puedan hacer efectivos sus créditos, por medio de lo que, conjeturablemente, podría asimilarse a un moderno procedimiento de verificación de los mismos.

\section{4) LA SUBROGACIÓN REAL}

La arquitectura conceptual del reparto tiene en la subrogación real un importante elemento ${ }^{21}$. En efecto, los juristas romanos no dejan de

butionem faciendam, ne ex alterius re merceve alii indemnes fiant, alii damnum sentiant [Pero si tuvo dos tiendas de un mismo negocio, y yo fui contador, por ejemplo, de la tienda que tuvo en Bucino y otro de la que tenía al otro lado del Tíber, considero muy justo que se hayan de computar por separado los distintos créditos, para que no cobren unos acreedores todo con las cosas o mercancías de otros, y salgan estos otros perjudicados].

20 Valiño (1967) 110.

21 Andrés SANTos (1997) 14, aunque adhiere a la idea que en el derecho romano la subrogación real fue operacionalizada en una gran variedad de soluciones propuestas por los juristas, admite que la formación consciente de un cierto "principio de subrogación real" tiene lugar solo a partir de la doctrina medieval. Es en efecto esta la que enuncia los aforismos "in universalibus, pretium succedit in locum rei, res in locum pretii, secus in particularibus" y "subrogatum sapit naturam eius in cuius locum subrogatum est". Sobre el desarrollo de este principio en el derecho medieval, Welle, Arno (1987) 'In universalibus pretium succedit in locum rei, res in locum pretii. Eine Untersuchung zur Entwicklungsgeschichte der dinglichen Surrogation bei Sondervermögen. Berlin: Duncker \& Humblot, 136 pp. 
recurrir a ella para establecer las bases de la contabilidad de los bienes a repartir y su utilidad no es menor, si se piensa que al estar integrada en un peculio, la merx peculiaris debe, a efectos del reparto, ser diferenciada de los restantes bienes del peculio, de haberlos. A modo de regla general, Ulpiano expresa en D. 14,4,5,11: Non autem totum peculium venit in tributum, sed id dumtaxat, quod ex ea merce est, sive merces manent sive pretium earum receptum conversumve est in peculium [No todo el peculio entra en el reparto, sino solo aquello que se encuentra por causa de aquella mercancía, sea porque permanecen en el peculio las mismas mercancías, sea porque se recibe o invierte en él lo recibido a cuenta de aquellas]. Desde luego, la expresión más clara de la operatividad de la subrogación real se manifiesta en el período "ex ea merce est - in peculium", por medio de la cual Ulpiano hace referencia a la mercancía, al precio de las $\operatorname{cosas}^{22}$ y a cualquier destino que se le haya dado, sea que se conserve en caja (receptum), sea que con él se hayan adquirido otros bienes, o bien, haya sido invertido (conversum). La idea del dinero conversum había sido ya abordada por Labeón, de acuerdo con la cita que de él hace Ulpiano, a fin de explicitar que los accesorios de la tienda, en cuanto hubiesen sido comprados con dinero proveniente de la merx deberían considerarse dentro de la contabilidad del reparto, como se aprecia en D. 14,4,5,13: Si praeter mercem servus iste in tabernam habeat instrumentum, an hoc quoque tribuatur? et Labeo ait et hoc tribui, et est aequissimum: plerumque enim hic apparatus ex merce est, immo semper. cetera tamen, quae extra haec in peculium habuit, non tribuentur, ut puta argentum habuit vel aurum, nisi si haec ex merce comparavit [Si a causa de la mercancía este esclavo tuviese accesorios en la tienda, ¿deben también estos entrar en el reparto? Labeón sostiene que también estos se reparten, lo que es muy justo, porque muchas veces e incluso siempre, este accesorio se compró con dinero proveniente de la mercancía. Con las demás cosas, en cambio, que tuvo aparte del peculio, no entrarán a reparto. Por ejemplo, si tuvo plata u oro, a menos que los haya comprado con la mercancía]. El mismo régimen que los accesorios siguen los esclavos que no han formado parte de la masa de bienes inicial, pero que han ingresado al peculio en virtud de la gestión de la mer $x^{23}$. Para Andrés Santos "la idea de subrogación real preside, pues, toda la explicación de Ulpiano en estas líneas: no es la voluntad del sujeto que negocia lo que establece el contenido de la masa de responsabilidad, sino criterios objetivos de imputación de derechos, particularmente la cláusula de la procedencia de los medios empleados para toda adquisición" 24 . A mayor abundamiento, Ulpiano, en D. 14,4,5,14 afirma: Item si mancipia

ANDRÉs SANTOS (1987) 202.

SuÁrez BLÁsquez (2013) 190.

Andrés Santos (1997) 203. A su turno, VAliño (1967) 112, reconoce igualmente a propósito de este texto la operatividad de la subrogación real. 
in negotiatione habuit ex merce parata, etiam haec tribuentur [Igualmente, si en la negociación hubiese esclavos adquiridos por la mercancía, también estos entran a reparto].

Un aspecto algo problemático, a propósito de este tema, se relaciona con aquellas mercaderías que subsisten en el peculio, y la razón para ello es que hay dos decisiones que parecen contradictorias. Un primer asunto se plantea a propósito de aquellas mercaderías que han sido adquiridas merced a un mutuo. Como se ha visto, la operatividad de la subrogación real no ofrece mayores dificultades cuando se trata del mismo capital. Pero los juristas prestan atención al supuesto en que el dinero con el que se adquieren los bienes ya no provienen del capital propio, sino de un préstamo. El problema es abordado por Ulpiano en D. 14,4,5,17: Plane si in eadem taberna merces deferebantur, licet hae quae exstent ex unius creditoris pecunia sint comparatae, dicendum erit omnes in tributum venire, nisi fuerint creditori pigneratae. [ $\mathrm{Si}$ en la misma tienda se vendían todas las mercancías, aunque las que quedan sin vender hayan sido compradas con dinero de un solo acreedor, se habrá de decir que todas habrán de computarse en el reparto, a no ser que hubiesen sido dadas en prenda al acreedor]. Las condiciones que plantea Ulpiano para admitir que las mercancías de las que trata este pasaje debieran entrar también a reparto, exigen alguna precisión. Un primer supuesto es que todas las mercancías eran vendidas en una misma tienda, lo que podría sugerir que se tratase de bienes fungibles, en el sentido de que la tienda albergase a todos los de un mismo tipo, como si, por ejemplo, vendiese trigo. Sin embargo, esta hipótesis habrá de ser precisada, según se verá más adelante. El segundo requisito se relaciona con el hecho de que una parte de la mercancía expuesta a la venta en la tienda hubiese sido adquirida con dinero de uno que ahora comparece como acreedor, es decir, de un mutuante. Este supuesto no produce ningún efecto respecto de la conjetura de la fungibilidad de los bienes. El tercer requisito consiste en que los bienes remanentes en la tienda correspondan exactamente a aquellos que se compraron con el mutuo dinerario de uno de los acreedores. Si volvemos la mirada al primer supuesto, ha quedado pendiente la conjetura acerca de la fungibilidad de las cosas adquiridas por mutuo. En efecto, no hay en el texto ningún indicio acerca de a qué clase de cosas hace referencia. Con todo, parece que hay que desechar que las cosas compradas con el mutuo fuesen fungibles con las demás cosas existentes en la tienda, por la sencilla razón de que, entonces, no sería posible diferenciarlas y, por lo mismo, no podría verificarse el tercer requisito. Por lo anterior, solo cabría la posibilidad de que se tratase, o bien de un género limitado (por ejemplo, de cierta cantidad de trigo en sacos de ciertas características que permitiera su diferenciación de otros), o bien que se tratase de bienes fungibles, pero no con relación a los demás bienes vendidos en la tienda. Por ejemplo, si con el mutuo se hubiese comprado miel, en tanto que las demás mercan- 
cías consistiesen en vino. Finalmente, podría tratarse de cosas específicas, de modo tal que no hubiese forma de confundirlas con otras mercancías.

El caso, como se observa, se centra en el problema de si el remanente, ya identificado como aquel adquirido gracias a un prestamo, debe o no integrar el reparto. Quien fuera mutuante figura como uno de los acreedores, por lo que, o bien el dinero mutuado no le fue restituido, o bien lo es por alguna otra causa. La respuesta de Ulpiano es afirmativa y, por consiguiente, esas mercancías habrán de entrar a reparto. La razón no debiese extrañarnos, pero vale la pena detenerse un momento. Por una parte, hemos visto que el edicto exige, para dar lugar al reparto, haya cosas en el peculio que se relacionen con las negociaciones para las cuales este se entrega. Si estas negociaciones deben recaer sobre la venta de distinta clase de bienes, la celebración de un mutuo para comprar y luego vender parece ser un operación admitida dentro de ellas. Por lo mismo, el mutuante no encuentra objeción alguna para participar del reparto. En cuanto al dinero mutuado, naturalmente se hace de quien lo recibe y, por consiguiente, se incorpora al peculio. Luego, si con él se adquieren bienes para la venta, entonces habrá que decir que tales mercancías se adquirieron con bienes del peculio y, por consiguiente se subrogaron a este. Por lo mismo, la decisión de Ulpiano no es extraña ni tampoco innovadora: tan solo es consecuencia de ciertas premisas: unas, las que se relacionan con el objeto de las negociaciones y otras, con las reglas de procedencia de la actio certi creditae pecuniae.

Un segundo aspecto del problema es el que plantea el mismo U1piano en D.14,4,5,18: Sed si dedi mercem meam vendendam et exstat, videamus, ne iniquum sit in tributum me vocari. et si quidem in creditum ei abiit, tributio locum habebit: enimvero si non abiit, quia res venditae non alias desinunt esse meae, quamvis vendidero; nisi aere soluto vel fideiussore dato vel alias satisfacto, dicendum erit vindicare me posse [Di mi mercancía para la venta y aún no se vende ¡es injusto convocarme al reparto <como acreedor >? Si, en efecto, lo dejé a crédito <el precio>, el reparto tendrá lugar; por el contrario, si no lo dejé a crédito, y ya que las cosas, aunque se hubiesen vendido, no se han hecho de otro, son mías, se habrá de decir que las puedo reivindicar, a menos que se pague su precio, o se otorgue fiador o bien se ha satisfecho de otra forma].

La pregunta que se formula tiene algunos presupuestos que es conveniente aclarar por separado. Un primer aspecto tiene que ver con que el remanente de mercancía corresponde a aquella que el dominus entregó al constituir el peculio y, por consiguiente, es de su propiedad. No es necesario avanzar sobre las circunstancias que rodean a esta situación, puesto que son asimilables a las que veíamos en el caso anterior. La pregunta que se hace Ulpiano es si en tal circunstancia resulta justo convocar al reparto al dominus: su respuesta la hace depender de las condiciones en que la mercadería fue entregada. La primera posibilidad es que se trate de una 
datio de mercaderías, de modo que el precio de venta de las mismas quede mutuado ${ }^{25}$, que es lo que se quiere significar con la expresión in creditum ei abiit. Esta modalidad exige, desde luego, que la venta se haya producido y el precio se haya recibido. Pero si, por el contrario, no se ha vendido, la mercadería que se dejó a crédito se exigirá mediante condictio, por cuanto se configuraría en tal caso una datio ob rem. La segunda posibilidad es que las mercaderías no hayan sido dejadas a crédito, sino que hayan sido aportadas al peculio. En este punto la situación descrita por Ulpiano dista mucho de ser clara. Por lo pronto, nuestro jurista concede la rei vindicatio al dueño de las cosas mientras estas no han sido enajenadas, aunque hayan sido vendidas. El pago del precio es tratado como un límite para la rei vindicaitio, y lo mismo puede decirse del otorgamiento de un fiador. Pero esta respuesta olvida la pregunta inicial. En esta se consultaba a qué título se presentaba el dominus, en el sentido de si era o no acreedor y sobre ello esta parte de la respuesta nada resuelve, porque en su calidad de dueño de las mercaderías podría concurrir en igualdad de condiciones que los que sí son acreedores. Pero es que, además, carece de toda lógica reivindicar las mercaderías si con ello perjudica a los demás acreedores.

\section{5) CONClusiones}

Como ha podido observarse, el procedimiento de insolvencia de la tributio de la merx peculiaris requirió de una configuración de cierta complejidad. Esto no debiera sorprender si se toma en cuenta que las cantidades repartidas y su proporción podían dar origen -junto a otros supuestos- a la sanción del dominus. La configuración a la que hacemos referencia se caracterizó por un cuidado trabajo de interpretación jurisprudencial, cuyo punto de partida fueron las cláusulas del edicto de tributoria actione. El producto de este trabajo interpretativo fue la elaboración de criterios tendientes, en primer término, a la determinación de los bienes a repartir (cláusulas quid contractum erit, eius rei nomine y ex ea merce) y, dentro de este mismo contexto, una definición acerca de lo que se entendería por merx. En segundo término, la jurisprudencia operacionalizó aquello que más tarde, desde época medieval, se conocería como el principio de la subrogación real. De este modo, la jurisprudencia estuvo en condiciones de esbozar y desarrollar las bases de un reparto que, como se dijo, estaba pergeñado de consecuencias.

25 El mismo Ulpiano trata esta clase de dación en D. 12,1,11 pr. 


\section{BIBLIOGRAFÍA CITADA}

Abatino, Barbara - Dari-Mattiacci, Giuseppe - Perotti, Enrico C. (2011) "Depersonalization of Business in Ancient Rome". Oxford Journal of Legal Studies vol. 31, No 2, pp. 365-389.

AndrÉs SANTOS, Francisco (1997) La subrogación real en los patrimonios especiales. Valladolid: Secretariado de Publicaciones e intercambio Científico de la Universidad de Valladolid, 317 pp.

Aubert, Jean Jacques (2013) “Dumtaxat de peculio': What's in a Peculium or Establishing the Extent of the Principal's Liability". En Du Plessis, Paul J. (editor). New Frontiers. Law and Society in the Roman World. Edinburgh: Edinburgh University Press, 246 pp.

Buti, Ignazio (1976) Studi sulla capacità patrimoniale dei servi. Napoli: Jovene, $240 \mathrm{pp}$.

Cerami, Pietro - Petrucci, Aldo (2010) Diritto commerciale romano. Profilo storico. $3^{\mathrm{a}}$ ed. Torino: Giappichelli, $337 \mathrm{pp}$.

Chiusi, Tiziana (1993) "Contributo allo studio dell'editto 'de tributoria actione”. Atti della Accademia Nazionale dei Lincei, serie IX, vol. III, fasc. 4, pp. $283-397$.

De Churruca, Juan (1991) "La quiebra de la banca del cristiano Calisto". Seminarios Complutenses de Derecho Romano, vol. 3, pp. 6186,

Di Porto, Andrea (1984) Impresa collettiva e schivavo 'manager' in Roma antica. Milano: Giuffrè, 407 pp.

Domingo, Rafael (1994-1995) "Recensión a Tiziana J. Chiusi, Contributo allo studio dell'editto de tributoria actione". Seminarios Complutenses de Derecho Romano vol. 5 (Suplemento), pp. 56-64.

Giachi, Cristina (2005) Studi su Sesto Pedio. Milano: Jovene, 601 pp.

Gimenez Barriocanal, Fernando (2003) La actividad económica en el Derecho romano. Análisis contable. Madrid: Dykinson, $454 \mathrm{pp}$.

Kirschenbaum, Aaron (1987) Sons, slaves and freedmen in Roman commerce. Jerusalem - Washington D.C.: The Magna Press, The Hebrew University, The Catholic University of America Press, 244 pp.

KNocH, Stefan (2005) Sklavenfürsorge im römischen Reich. Formen und Motive. Hildesheim - Zürich - New York: Georg Olms, 338 pp., p. 178 ss.

Kroppenberg, Inge (2001) Die Insolvenz im klassichen römischen Recht. Wien-Köln: Böhlau, 526 pp.

Lazo, Patricio (2010) "El contexto dogmático de la 'par condicio creditorum' en el derecho romano". Revista de Derecho, Universidad Católica del Norte, vol. 17, No 2, pp. 79-97. 
LAzo, Patricio (2013) "La 'merx peculiaris' como patrimonio especial". Revista de Estudios Histórico-Jurídicos, vol. 35, pp. 179-191.

Lenel, Otto (1927) Das Edictum Perpetuum. 3a ed. Leipzig: Tauchnitz, 579 pp., p. 271.

Petrucci, Aldo (1991) Mensam exercere. Studi sullimpresa finanziaria romana. Napoli: Jovene, 418 pp.

Suárez Blásquez, Guillermo (2010) “El peculio como ente autónomo y matriz de la 'merx peculiaris". Revista de Estudios Histórico-Jurídicos, vol. 32, pp. 119-125.

Suárez Blázquez, Guillermo (2013) “Concurso mercantil de acreedores: 'vocatio in tributum'. La acción tributoria frente al dueño (empresario corrupto)". Revista de Derecho, Universidad del Norte, vol. 39, pp. 173-197, p. 189.

VAliño, Emilio (1967) "La 'actio tributoria". Studia et Documenta Historiae et Iuris, vol. 33, pp. 103-128.

Welle, Arno (1987) 'In universalibus pretium succedit in locum rei, res in locum pretii'. Eine Untersuchung zur Entwicklungsgeschichte der dinglichen Surrogation bei Sondervermögen. Berlin: Duncker \& Humblot, 136 pp. 